LAUR-98-5276

\title{
Apparent horizons in simplicial Brill wave initial data
}

\author{
Adrian P. Gentle田, Daniel E. Holz and Warner A. Miller \\ Theoretical Division (T-6, MS B288), \\ Los Alamos National Laboratory, Los Alamos, NM 87545, USA
}

John A. Wheeler

Physics Department,

Princeton University, Princeton, NJ 08544, USA

\begin{abstract}
We construct initial data for a particular class of Brill wave metrics using Regge calculus, and compare the results to a corresponding continuum solution, finding excellent agreement. We then search for trapped surfaces in both sets of initial data, and provide an independent verification of the existence of an apparent horizon once a critical gravitational wave amplitude is passed. Our estimate of this critical value, using both the Regge and continuum solutions, supports other recent findings.
\end{abstract}

PACS numbers: 04.20.-q, 04.60.Nc, 04.25.Dm

\section{Introduction}

Regge calculus [1] is used to re-examine a particular class of Brill wave spacetimes [2] investigated by Miyama [3], and more recently, Holz et al 沟] and Alcubierre et al [5]. In particular, we study the formation of apparent horizons within the simplicial initial data, and confirm recent findings regarding the appearance of such horizons [5].

Brill wave initial data was first constructed using Regge calculus by Dubal [6], although we show elsewhere [7] that his procedure is unable to capture the full structure of complex axisymmetric initial data sets. By using an improved lattice (which is described in full in Ref. [7), we are able to accurately reproduce the continuum solution, and find apparent horizons in excellent agreement with recent two and three-dimensional calculations [5].

We proceed as follows. In the next section, we briefly survey the approach taken by Brill [2] in the construction of gravitational wave initial data. In section 3 we describe the axisymmetric lattice used to construct Brill waves using Regge calculus. In section 1 we describe the method used to find apparent horizons at a moment of time symmetry, and in section 5 we investigate the appearance of apparent horizons in the Brill wave space as the wave amplitude is increased.

$\ddagger$ Permanent Address: Department of Mathematics and Statistics, Monash University, Clayton, Victoria 3168, Australia. Email address adrian@lanl.gov. 


\begin{tabular}{|c|c|c|c|c|}
\hline $\mathrm{a}$ & $M_{R}$ & $M_{C}$ & $M$ (Holz et al $)$ & $M$ (Alcubierre et al $)$ \\
\hline 1 & $3.38 \times 10^{-2}$ & $3.38 \times 10^{-2}$ & $3.40 \times 10^{-2}$ & $3.38 \times 10^{-2}$ \\
2 & $1.26 \times 10^{-1}$ & $1.26 \times 10^{-1}$ & $1.26 \times 10^{-1}$ & $1.27 \times 10^{-1}$ \\
5 & $6.98 \times 10^{-1}$ & $6.98 \times 10^{-1}$ & $6.96 \times 10^{-1}$ & $7.00 \times 10^{-1}$ \\
10 & 2.91 & 2.91 & 2.91 & 2.91 \\
12 & 4.67 & 4.67 & 4.67 & 4.68 \\
\hline
\end{tabular}

Table 1. Mass estimates for the Regge $\left(M_{R}\right)$ and continuum solutions $\left(M_{C}\right)$. The mass is calculated from the decay of $\psi$ in the asvmptotic region. We also show the results of previous calculations by Holz et al [4] and Alcubierre et al [ which are in excellent agreement. All our results were calculated on a $600 \times 600$ grid.

\section{The continuum model}

We examine the class of axisymmetric vacuum spacetimes containing gravitational waves which admit a moment of time symmetry originally studied by Brill [2]. Brill [2] relaxed the conformal flatness criteria and introduced a spatial metric of the form

$$
d s^{2}=\psi^{4}\left\{e^{2 q}\left(d \rho^{2}+d z^{2}\right)+\rho^{2} d \phi^{2}\right\},
$$

where the arbitrary function $q(\rho, z)$ can be considered the distribution of gravitational wave amplitude [8]. In order to obtain an axisymmetric solution which is reflection symmetric about $z=0$, we require $q_{, \rho}=0$ along $z=0$ and $q=q_{, z}=0$ on $\rho=0$, together with the condition that $q$ falls off sufficiently quickly (at least $r^{-2}$ ) in the asymptotic zone. Apart from these boundary conditions, $q(\rho, z)$ is arbitrary.

At a moment of time symmetry the constraint equations of General Relativity reduce to the single condition

$$
R=0 \text {, }
$$

where $R$ is the three-dimensional Ricci scalar. With Brill's choice of background metric, the Hamiltonian constraint (2) takes the form

$$
\nabla^{2} \psi=-\frac{\psi}{4}\left(\frac{\partial^{2} q}{\partial \rho^{2}}+\frac{\partial^{2} q}{\partial z^{2}}\right)
$$

which is solved for $\psi(\rho, z)$ once $q(\rho, z)$ is given. In the current work we adopt the form of $q(\rho, z)$ chosen by Miyama [3],

$$
q=a \rho^{2} e^{-r^{2}}
$$

where $r^{2}=\rho^{2}+z^{2}$, and the wave amplitude $a$ is a free parameter.

Since we require an axisymmetric, asymptotically flat solution which is reflection symmetric on the $z=0$ axis, we enforce the boundary conditions

$$
\frac{\partial \psi}{\partial \rho}(0, z)=0, \quad \frac{\partial \psi}{\partial z}(\rho, 0)=0,
$$

on $\psi$, together with a Robin condition at the outer boundary,

$$
\frac{\partial \psi}{\partial r}=\frac{1-\psi}{r},
$$

which ensures that the hypersurface is asymptotically Euclidean.

The solution for $\psi$ is calculated using these boundary conditions together with a second order accurate finite difference approximation to equation (3). 


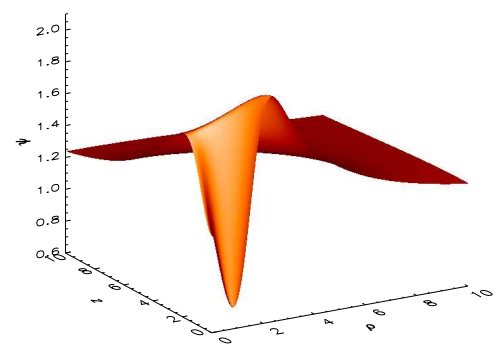

(a)

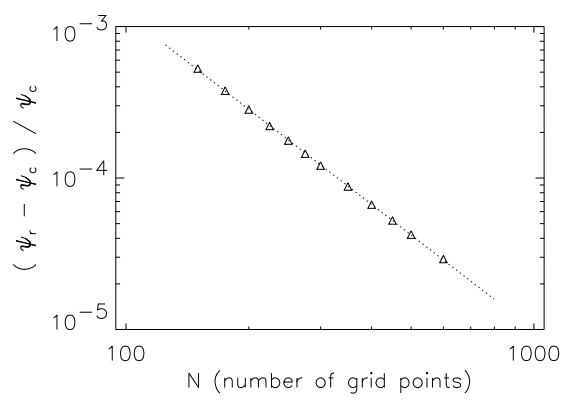

(b)

Figure 1. (a) The conformal factor for $a=12$ calculated using the Regge lattice with $600 \times 600$ vertices. The solution is shown on the first quadrant of the $\rho-z$ plane, with the outer boundaries placed at $\rho=z=20$. (b) The fractional difference, averaged pointwise across the domain, between the simplicial $\left(\psi_{r}\right)$ and continuum $\left(\psi_{c}\right)$ solutions for the conformal factor on an $N \times N$ grid.

\section{The Regge calculus model}

In this section we will briefly outline the axisymmetric tetrahedral lattice used to construct Regge initial data. Full details of the approach described here may be found elsewhere [7].

The simplicial equivalent of the initial value constraint at a moment of time symmetry, equation (2), has been given by Wheeler [8],

$$
\sum_{b} L_{a b} \epsilon_{a b}=0 .
$$

This is the three-dimensional simplicial equivalent of $R=0$ at a vertex $a$, where $L_{a b}$ is the lattice edge joining vertex $a$ to vertex $b$, and $\epsilon_{a b}$ is the deficit angle (product of the curvature on the edge by the area dual to the edge) about $L_{a b}$. The summation is over all edges $L_{a b}$ which meet at vertex $a$.

We mirror the continuum approach and perform a conformal decomposition on the lattice edges, since they are the simplicial equivalent of the metric. This gives

$$
L_{a b}=\psi_{a b}^{2} \bar{L}_{a b}
$$

where $\bar{L}_{a b}$ is a freely chosen base edge length. It is most natural to define the simplicial conformal factor on the vertices of the lattice, such that $\psi_{a}$ is the conformal factor at vertex $a$. The conformal factor $\psi_{a b}$ acting on the edge $L_{a b}$ is then defined using a centred, second order approximation,

$$
\psi_{a b}=\frac{1}{2}\left(\psi_{a}+\psi_{b}\right) .
$$

We assign the base edges $\bar{L}_{a b}$ such that the lattice is aligned with the co-ordinate grids of the continuum approach. Figure 2 indicates how we subdivide each block in the co-ordinate grid to obtain a tetrahedral three geometry. Given the full set of physical edge lengths in this lattice, we calculate the deficit angles (curvatures) about each edge, and write down the simplicial initial value constraint, equation (7). This equation is then solved, together with suitable boundary conditions, for the conformal factor at each vertex of the lattice. 


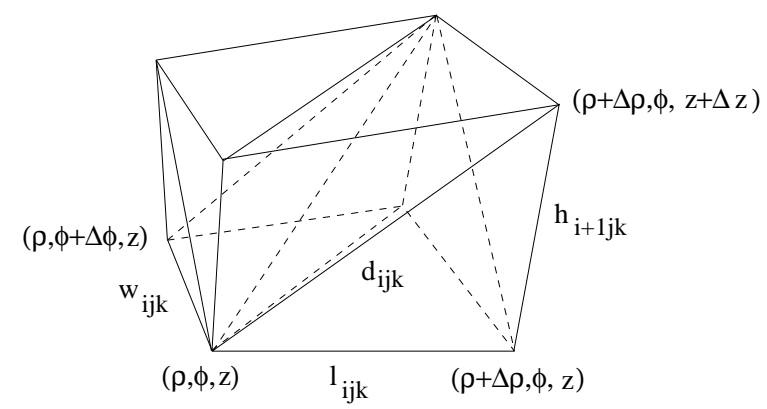

Figure 2. A portion of the simplicial three-lattice, which shows how each coordinate block is subdivided into six tetrahedra. After calculating the geometric quantities for this lattice, the limit $\Delta \phi \rightarrow 0$ is taken, in order to obtain a discretization suitable for constructing axisymmetric initial data.

To obtain a purely axisymmetric lattice, we take the limit as $\Delta \phi$ tends to zero, while demanding that each block is symmetric about $\phi+\frac{1}{2} \Delta \phi$. Retaining only the leading order terms of equation (7) in this limit yields the axisymmetric simplicial initial value equation at a moment of time symmetry. Full details of this calculation and the resulting equations may be found elsewhere [7].

By aligning the lattice with a cylindrical polar co-ordinate system, we may use the form of the continuum base metric, equation (11), to specify the base edges in the lattice. Combining this with the conformal decomposition described above, and noting that after the limit has been taken there are only three non-zero edges per vertex, the physical lattice edges take the form

$$
\begin{aligned}
l_{i k} & =\left[\psi^{2} e^{q}\right]_{i+\frac{1}{2}, k} \Delta \rho_{i} \\
d_{i k} & =\left[\psi^{2} e^{q}\right]_{i+\frac{1}{2} k+\frac{1}{2}}\left(\Delta \rho_{i}^{2}+\Delta z_{k}^{2}\right)^{1 / 2} \\
h_{i k} & =\left[\psi^{2} e^{q}\right]_{i, k+\frac{1}{2}} \Delta z_{k}
\end{aligned}
$$

where all expressions are centred on the edge, and we have neglected the redundant index along the $\phi$ axis.

We are now able to calculate the simplicial initial data by solving equation (7) for the conformal factor $\psi$. We use the same boundary conditions as before, applied along the $\rho=0$ and $z=0$ boundaries using power series expansions into the domain.

The solution for $\psi$ calculated using the Regge lattice, together with the boundary conditions described in section 2, is shows in figure 1 1 a. The solution displays excellent agreement with the previous calculations of Holz et al and Alcubierre et al [4, 5], and the continuum model developed in section 2. Figure $11 b$ contains a rigorous convergence analysis which demonstrates that the Regge solution converges to the numerical continuum solution as the second power of the lattice spacing. For further details, we refer the reader to Gentle [7].

Both solutions display good agreement with the previous calculations of both Holz et al [4] and Alcubierre et al [5]. Table 1 contains estimates of the mass of the Brill waves, calculated using a least squares fit of the function

$$
\psi_{a}=1+\frac{M}{2 r}
$$


to $\psi$ in the asymptotic zone. The mass $M$ is found to converge rapidly with radius.

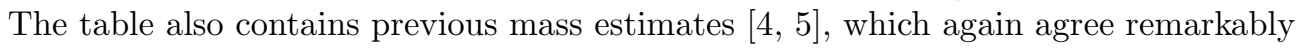
well with the current calculations.

\section{Trapped surfaces}

Our goal in the remainder of this paper is to locate any apparent horizon which exists in the Brill wave data. Apparent horizons are defined [9] as the outermost trapped surface on the spatial hypersurface. That is, the outermost closed, spacelike twosurface on which the divergence of outgoing null rays is zero. At a moment of time symmetry, this is equivalent to the outermost closed spacelike surface with extremal surface area.

In order to construct the trapped surfaces we utilize the technique employed by Holz et al 画, where the search for an extremal two-surface was shown to be equivalent to the construction of geodesics in a certain two-dimensional space.

Let us consider a path $s(\rho, z)$ in the first quadrant of the $\rho-z$ plane, which forms a closed surface when rotated about the $z$ and $\rho$ axes. The area of this surface of rotation is

$$
S=\int_{P_{0}}^{P_{1}} 2 \pi \psi^{4} e^{q} \rho\left(d \rho^{2}+d z^{2}\right)^{1 / 2},
$$

and the criteria for a trapped surface is that this area is extremal, $\delta S=0$. It is straightforward to show that this extremization is mathematically equivalent to finding the geodesics of the metric [n]

$$
d s^{2}=4 \pi^{2} \psi^{8} \rho^{2} e^{2 q}\left(d \rho^{2}+d z^{2}\right),
$$

subject to appropriate boundary conditions. If the geodesics are parameterized by $\lambda$, the appropriate boundary conditions are

$$
\begin{aligned}
& \dot{\rho}\left(\lambda_{0}\right)=0 \quad \text { and } \quad z\left(\lambda_{0}\right)=0, \\
& \dot{z}\left(\lambda_{1}\right)=0 \quad \text { and } \quad \rho\left(\lambda_{1}\right)=0,
\end{aligned}
$$

where $\lambda_{0}$ and $\lambda_{1}$ are the endpoints of the curve, and $\dot{\rho}$ represents differentiation with respect to $\lambda$. These boundary conditions ensure that the curves are continuously differentiable across the axes.

The trapped surfaces are constructed using a shooting method. Given a value of $\rho\left(\lambda_{0}\right)$, the geodesic is traced to $\lambda_{1}$, where we check how closely the boundary condition is satisfied. Once a pair of $\rho\left(\lambda_{0}\right)$ values have been found which bound the condition $\dot{z}\left(\lambda_{1}\right)=0$, the bisection method is used to converge on the trapped surface.

It would be interesting to construct an algorithm for finding trapped surfaces entirely within the framework of Regge calculus. An obvious approach at a moment of time symmetry would be to implement a direct search algorithm which minimizes the simplicial surface area of a topological two-sphere. For the purposes of the current work, we have assumed that the simplicial lattice corresponds to a section of a differentiable manifold, upon which we can apply the ideas outlined above. We use the conformal factor $\psi_{r}$, calculated using Regge calculus, to construct the geodesics of metric (12), and hence construct trapped surfaces within the lattice. 


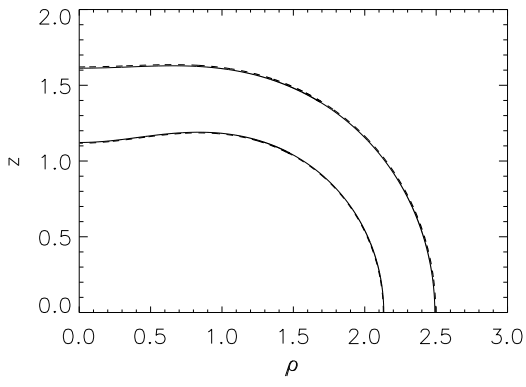

(a)

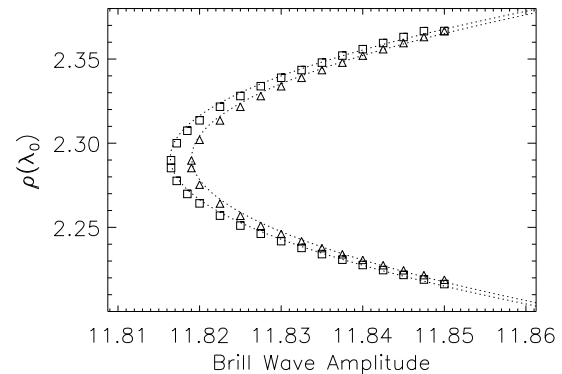

(b)

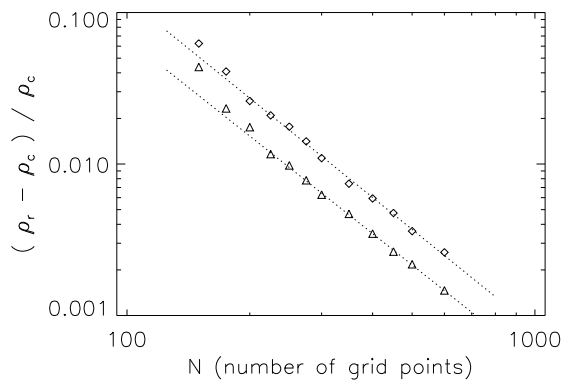

(c)

Figure 3. (a) Trapped surfaces for a Brill wave with amplitude $a=12$ at a moment of time symmetry. The outermost trapped surface is the apparent horizon. The calculations were performed using both the Regge (solid lines) and continuum (dashed lines) solutions on a $600 \times 600$ grid, and are in excellent agreement. (b) The point $\rho\left(\lambda_{0}\right)$ where the inner and outer trapped surfaces meet the $\rho$-axis is plotted as a function of wave amplitude, close to the critical amplitude $a_{*}$. The quadratic fit suggests that $a_{*} \approx 11.819$ in the Regge solution (triangles), and $a_{*} \approx 11.816$ in the continuum initial data (squares). (c) Convergence of the trapped surfaces calculated using the two sets of initial data. For a Brill wave amplitude of $a=12$, we show the fractional difference between the Regge and continuum values of $\rho\left(\lambda_{0}\right)$ as the grid resolution is increased. Both the inner and outer trapped surfaces are shown. The two solutions converge as the second power of the grid spacing.

\section{Apparent horizons}

Using the technique outlined in section 4 , we now search for apparent horizons in the Brill wave initial data constructed in sections 2 and 3

We find that in both the Regge and continuum solutions an apparent horizon first appears at a critical amplitude $a_{*}$, which lies in the range $a_{*} \in[11.81,11.82]$. As the amplitude $a$ is increased above this critical value, two trapped surfaces are present, and these gradually separate. These results are in complete agreement with the recent calculations of Alcubierre et al [5].

Figure 3a shows a projection of the trapped surfaces found for a wave amplitude of $a=12$, calculated using both the continuum and Regge solutions. There is a slight difference between the surfaces calculated using these two independent sets of initial 
data, but the discrepancy is found to reduce as the second power of the grid spacing. In figure $3 b$, we show the position $\rho\left(\lambda_{0}\right)$ of both trapped surfaces along the $\rho$-axis as a function of the Brill wave amplitude. The quadratic fit to this data allows us to improve the estimate of the critical wave amplitude at which an apparent horizon first forms. We estimate that $a_{*} \approx 11.819$ for the simplicial solution, and $a_{*} \approx 11.816$ for the solution to the continuum equation. Both solutions predict that the single horizon at $a=a_{*}$ passes through $\rho\left(\lambda_{0}\right) \approx 2.291$.

The value of the critical amplitude $a_{*}$, at which an apparent horizon first forms, differs from that obtained in an earlier calculation by some of us 断. The discrepancy in the earlier work was due to the relatively weak convergence criteria used for the trapped surface finder. Qualitatively, the previous work agrees with both the present calculations and those of Alcubierre et al [5].

\section{Future work}

We have used Regge calculus to confirm recent numerical findings regarding the formation of apparent horizons in a particular class of Brill wave spacetimes, while demonstrating that Regge calculus provides an alternative and competitive technique for use in numerical relativity.

Brill wave spacetimes provide a challenging test-bed for the future development of simplicial gravity. Work is currently underway on the development of a simplicial trapped surface finder for Regge calculus, as well as the time evolution of the initial data constructed here, in both $(2+1)$ and $(3+1)$-dimensions.

\section{Acknowledgments}

We gratefully acknowledge support from a Los Alamos National Laboratory LDRD Grant. One of us (APG) acknowledges support from the Sir James McNeill Foundation at Monash University, and from the Center for Nonlinear Studies.

\section{References}

[1] T. Regge, Nuovo Cimento , 19, 558-71 (1961).

[2] D. R. Brill, Ann. Phys., 7, 466-83 (1959).

[3] S. M. Miyama, Prog. Theor. Phys., 65, 894-909 (1981).

[4] D. E. Holz, W. A. Miller, M. Wakano and J. A. Wheeler, in Directions in General Relativity: Proceedings of the 1993 International Symposium, Maryland; Papers in Honour of Dieter Brill, eds. B. L. Hu and T. Jacobson (Cambridge Uni. Press, 1993).

[5] M. Alcubierre, S. Brandt, B. Brügmann, C. Gundlach, J. Masso, E. Seidel and P. Walker, gr-qc/9809004.

[6] M. R. Dubal, Class. Quantum Grav. , 6, 141-55 (1989).

[7] A. P. Gentle, To appear.

[8] J. A. Wheeler, in Relativity, Groups and Topology, ed. C. DeWitt and B. DeWitt (Blackie and Son Ltd., 1964)

[9] S. W. Hawking and G. F. R. Ellis, The large scale structure of space-time, Cambridge University Press, Cambridge, 1973. 